\section{US cancer trends}

SIR - Your article on the US Surgeon General's report The Health Consequences of Smoking: Cancer 1982 (Nature 4 March, p.4) again draws attention to the importance of smoking as a cause of cancer. The "remarkable increases in mortality from smoking-related cancers", a widely recognized phenomenon, is usefully reiterated by the Surgeon General. The concurrent decline in the death rate from all non-smoking-related cancers, also undisputed, is reported in the Nature news item. We would like to point out a misinterpretation of the reasons for the decline in cancers not related to smoking which has followed publication of the report.

The Surgeon General cites "improvements in survival rates for some cancers through earlier or better diagnosis and treatment" during the period of steeply increasing smoking-related cancer mortality rates. As had been well publicized, improvements have been made in the treatment of Hodgkin's disease, some childhood leukaemias, testicular cancer, and a few others. In contrast, however, improvements in survival of people afflicted with solid tumours, those responsible for the vast majority of cancer deaths, have been small. The improvements in survival, while important, have had little effect on overall mortality from non-smoking-related cancers.

The major contributions to reduced nonrespiratory cancer mortality are the falling mortality rates for stomach and uterine cancers. These declines result not from improved survival, but from declining incidence rates for these cancers ${ }^{1}$.

Stomach cancer rates are now decreasing throughout the developed world. In the United States the trend continues downwards throughout middle age, suggesting that as younger people age, they will have rates lower than those of their parents' generation. Stomach cancer mortality rates in the United States are now among the lowest in the world. Early detection and improved treatment has had little impact on the mortality rates. There has been practically no improvement in survival of stomach cancer patients since 1950 .

No single explanation adequately explains the decrease in stomach cancer incidence, but several factors have been suggested as contributors: modern techniques of food preparation and storage, increased consumption of green vegetables, fruits, antioxidants (as food preservatives) and increased milk intake.

The long downward trend in cervical cancer, which began at least 40 years ago, is the chief reason for the large, steady decrease in female non-respiratory cancer death rates over the past half century. The decline began long before the "Pap test" for early detection of cancer of the cervix became widespread. The causes of this substantial improvement are not fully understood, though it appears to have accompanied general improvements in public health and living standards.

The Nature news article cites improvements in survival of prostatic, colorectal and breast cancer patients as contributing to reductions in non-smoking-related cancer mortality. In fact, mortality rates for these three cancers have remained nearly constant since 1950 .

While the contemporary declines in mortality from stomach and uterine cancers appear to be fortuitous concomitants of modern life, additional major reductions in cancer rates can be achieved with present knowledge. The evidence indicting smoking provides the greatest unrealized potential for reducing the impact of cancer.

Michael. Gougih Hellen Gelband

Office of Technology Assessment, US Congress,

Washington D.C., USA

1. Assessment of Technologies for Determining Cancer Risks from the Environment (Office of Technology Assessment, US Congress, 1981).

\section{Anthrax island}

SIR - We would like to deal with a number of points raised by Dr Sterne (Nature 4 February, p.362) regarding our paper on anthrax contamination on Gruinard Island ${ }^{1}$.

The main object of the 1979 survey of Gruinard Island was to determine whether or not the whole island was grossly contaminated with Bacillus anthracis. We have shown that it is not, but that the organism is found in detectable numbers in a small area.

We believe that Dr Sterne has misunderstood our view about Van Ness's assumption that $B$. anthracis proliferates in the soil under certain conditions ${ }^{2}$. We stated in our paper that we did not believe such conditions existed on Gruinard Island. The present extent of the contamination was probably due solely to the wartime trials with some slight redistribution of the spores by wind and rain.

We agree with Dr Sterne's statement that he failed to recover $\boldsymbol{B}$. anthracis from cadavers of cattle buried as little as three months previously because it is known that only spores survive for any length of time. The vegetative bacilli only sporulate in the presence of oxygen $^{3,4}$, and veterinary officers dealing with suspected cases of anthrax do not normally open the body cavity thus ensuring that oxygen is excluded.

We have shown that if blood is added to contaminated soil from Gruinard Island the spores will germinate and proliferate. The numbers of indigenous soild bacteria did increase as a result of this procedure but the selective medium allowed us to obtain accurate counts of $\boldsymbol{B}$. anthracis colonies. Dr Sterne rightly states that there is no experimental evidence that $B$. anthracis does multiply in the soil but what we have tried to show is that the possibility of proliferation in the presence of nutrients does exist and should not be entirely disregarded.

\section{R.J. Manchee, M.G. Broster,} R.M. Henstridge, A.J. Stagi \& J. MeLling

Chemical Defence Establishment and PHLS

Centre for Applied Microbiology and Research, Porton Down, Wiltshire, UK

1. Manchee, R.J., Broster, M.G., Melling, J., Henstridge, R.M. \& Stage. A.J. Nature 294, 254-255 (1981). 2. Van Ness, G.B. Science 72, 1303-1307 (1971).

3. Marsh, J. in Newsome's Sheep Diseases, p. 3 (1973).

3. Marsh, J. in Newsome's Sheep Diseases, p.3 (1973). p.387 (1967)

\section{Improbable stuff}

SIR - That the information content of terrestrial biology is truly prodigious ${ }^{1}$ seems generally agreed. Hoyle and Wickramasinghe ${ }^{2}$ avoid the problem of assessing repetitiveness and irrelevancy in the DNA (Nature 1 October 1981, p.333) by starting from the fact that 2,000 enzymes are common to all life. Each enzyme has a distinct "backbone" of, say, a dozen amino acids together with a specific active site which catalyses a particular biochemical reaction and is determined by,say, six further amino acids. It is presumed that these common enzymes determine a minimum information content in biological DNA.

Taking the twenty biological amino acids, one can compute the number of different combinations of Boltzmann's ${ }^{3}$

"permutability" $P$ of enzyme-like chains: for 2,000 18-link chains $P$ would be $\sim 10^{40,000}$, for 20,000 18-link chains containing the 2,000 specific ones it would be $-10^{26,000}$, while for 2,0006 -link chains it would be $\sim 10^{13,000}$. In choosing the number of 6 to characterize the active site, Rout (Nature 18 March 1982, p.192) discounted the "backbone" but more importantly forgot that activities such as energy release from sugars or accurate DNA replication require sequences of particular enzymes catalysing reactions in a specific order.

Weighting the amino acids according to some expected abundances modifies the numbers but an index of the order of 10,000 is inescapable - that is, a probability of $1: 10^{10,000}$ against finding the present enzyme system in a particular sample at a particular time as a chance arrangement. To demonstrate the immensity of such numbers, note that the product of the number of bacterium-sized drops $\left(10^{-13} \mathrm{~cm}^{3}\right)$ in the upper 1 metre of the Earth's surface water and the number of milliseconds in 4 billion years is $10^{54}$. Alternatively, the number of UV photons over $4 \mathrm{eV}$ (capable of breaking and rearranging a chain) arriving through the Earth's history is $10^{49}$. All Earth-like planets in the Galaxy throughout its lifetime would give "only" $10^{78}$ or $10^{73}$.

It is probable that some simple selfreplicating systems exist that are viable in restricted environments, if only because present biology can prosper in extreme conditions and utilize a wide variety of exothermic chemical transformations ${ }^{2}$. So life might have started from a very simple biology, arising by chance on the primitive Earth. Experimental demonstration of a practical system is not inconceivably remote ${ }^{4}$.

But why don't geocentric evolutionists (Nature 18 March 1982, p.198) face up to the awkward questions: where are all the simpler intermediate biologies - surely some should have had advantages over our unnecessarily rich biology and have left clear traces? And why should bacteria be adaptive to nonterrestrial environments and in particular be capable of space travel?

Max Wal.i.is

University College Cardiff, UK

1. Cairns-Snith, A. G. J. theor. Biol. 10,53 (1965)

2. Hoyle, F. \& Wick ramasinghe, N. C. Evolution from Space (Dent, L.ondon, 1981).

3. Boilzmann, L.. Weiner Ber. 76,373 (1877).

4. Eigen, M., Gardiner, W., Schuster, P. \& W inckler Oswatitsch, R. Scient. Am. 182, 78 (1981). 